\title{
Anatomical studies on the cranial and caudal mesenteric arteries of the Barbados Black Belly sheep
}

\author{
MOHAMED, R. ${ }^{1,2}$ \\ ${ }^{1}$ Department of Basic Veterinary Sciences, School of Veterinary Medicine, Faculty of Medical Sciences, \\ The University of West Indies, Trinidad and Tobago \\ ${ }^{2}$ Anatomy and Embryology Department, Faculty of Veterinary Medicine, Beni Suef University, Egypt \\ *E-mail: reda.mohamed@sta.uwi.edu
}

\begin{abstract}
Introduction: The Barbados Black Belly is a breed of domestic sheep in the Caribbean island of Trinidad. Anatomical studies on the cranial and caudal mesenteric arteries are necessary to know the pattern of its blood supply to gain information in benefit of experimental surgery. Materials and Methods: The thoracic part of the aorta of five sheep was injected with red latex. Careful gross dissection of the cranial and caudal mesenteric arteries was performed either after embedding in 10\% formalin solution for 2-3 days. Results: The cranial mesenteric artery originated from the abdominal aorta, caudally to the celiac trunk, giving caudal duodenal artery, jejunal arteries, ileal arteries, ileocolic artery and middle colic artery. The caudal mesenteric artery arises from the aorta, cranially to the external iliac arteries, originating the left colic and cranial rectal arteries. Conclusion: cranial and caudal mesenteric arteries supplied the small and large intestine of the Barbados Black Belly sheep except caudal part of the large intestine which were supplied by the middle and caudal rectal arteries.
\end{abstract}

Keywords: anatomy, mesenteric arteries, intestine, sheep.

\section{Introduction}

The Barbados Black Belly is a breed of domestic sheep in the Caribbean island of Trinidad. Both male and female have no horns. This breed is raised primarily for meat. It combines the rare attributes of adaption to the environment and high reproductive efficiency, which account for their average of two lambs per litter and an average lambing interval of eight to nine months. Considering that the anatomical knowledge is necessary to understand the biological functions. The mesenteric arteries are responsible for the blood supply to the small and large intestine of reptiles, birds and mammals, where the digested nutrients are absorbed the epithelium and transferred to the circulation. Anatomical studies on the cranial and caudal mesenteric arteries of the Barbados Black Belly sheep are necessary to know the pattern of its blood supply to gain information in benefit of experimental surgery, pharmacology and toxicology which can be applied to domestic. Therefore, the present investigation aimed to describe fully the branches of these arteries, regarding the origin, course and organ supplying.

\section{Materials and Methods}

In this study, 5 adult apparently healthy Black Belly sheep of different ages and both sexes ranging from $20-30 \mathrm{~kg}$. body weight. The animals were collected from the school of veterinary medicine, Trinidad and Tobago. General anaesthesia was induced by xylazine (Rompun) $0.5 \mathrm{mg} / \mathrm{kg}$ and cyclohexanone (Ketalar) $10 \mathrm{mg}$ per kg body weight intramuscularly. Immediately after slaughtering, the abdominal cavity was opened by making a longitudinal incision was made in the midventral line of the abdominal wall starting from the xiphoid cartilage of the sternum till the anus. Then red latex was injected via the thoracic aorta just prior to its passage through the hiatus aorticus of the diaphragm. Careful gross dissection of the cranial and caudal mesenteric arteries was performed either after embedding in $10 \%$ formalin solution for 2-3 days. Dissected arteries were photographed. The nomenclature employed in this study was in accordance with that of the Nomina Anatomica Veterinaria (INTERNATIONAL..., 2005) and the available literatures whenever possible.

\section{Results}

\subsection{Cranial mesenteric artery}

The cranial mesenteric artery (Figures 1, 2, 3 and 4) originated separately from the ventral aspect of the abdominal aorta caudal to the origin of the celiac artery. It entered the mesentery giving caudal duodenal artery, jejunal arteries, ileal arteries, ileocolic artery and middle colic artery along its course and it terminated at the terminal part of the jejunum by its division into the last two jejunal arteries (Figure 3 ).

\subsection{Caudal duodenal artery}

The caudal duodenal artery (Figure 1) was the first branch of the cranial mesenteric artery. It passed caudally within the mesoduodenum giving off duodenal branches towards the mesenteric border of the ascending limb of the duodenum.

\subsection{Jejunal arteries}

The jejunal arteries (Figures 1, 2 and 3) were formed of numerus branches, distributed all over the jejunum. They arose directly from the cranial convex part of the cranial mesenteric artery. The last two jejunal arteries were represented the termination of the cranial mesenteric artery Each jejunal artery passed within the mesentery towards the mesenteric border of 


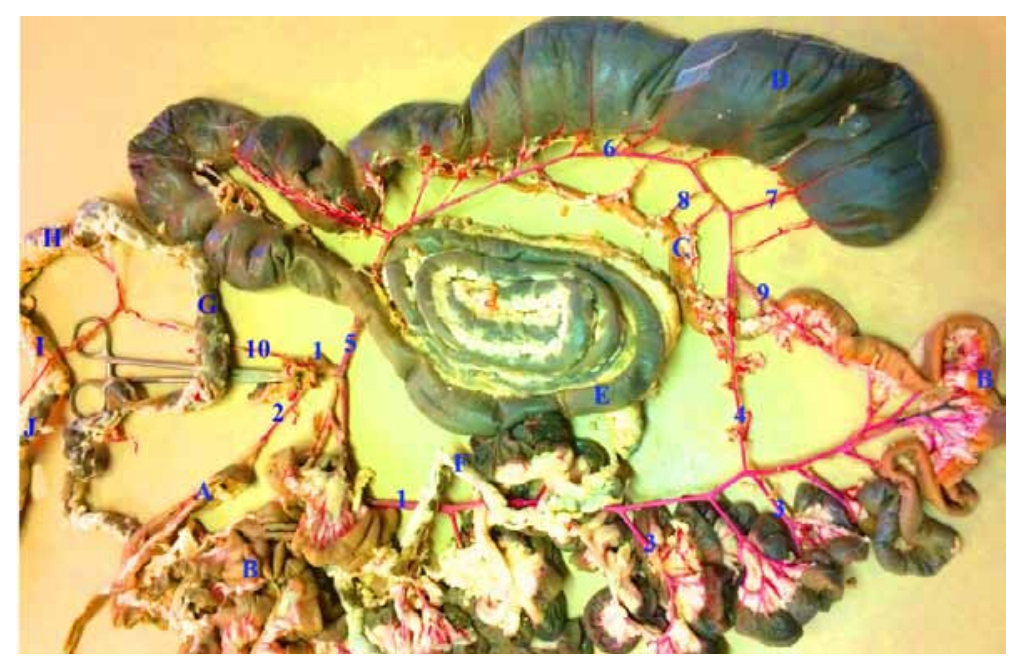

Figure 1. A photograph showing the course and distribution of the cranial mesenteric artery of the sheep; (A) Duodenum; (B) Jejunum; (C) Ileum; (D) Cecum; (E) Centripetal gyri; (F) The most outer centrifugal gyrus; (G) Dorsal gyrus; (H) Ventral gyrus; (I) Transverse colon; (J) Descend colon; (1) Cranial mesenteric artery; (2) Caudal duodenal artery; (3) Jejunal arteries; (4) Ileal arteries; (5) Ileocolic artery; (6) Cecal artery; (7) Cecal branches; (8) Antimesenteric ileal branches; (9) Antimesenteric ileal artery; (10) Middle colic artery.

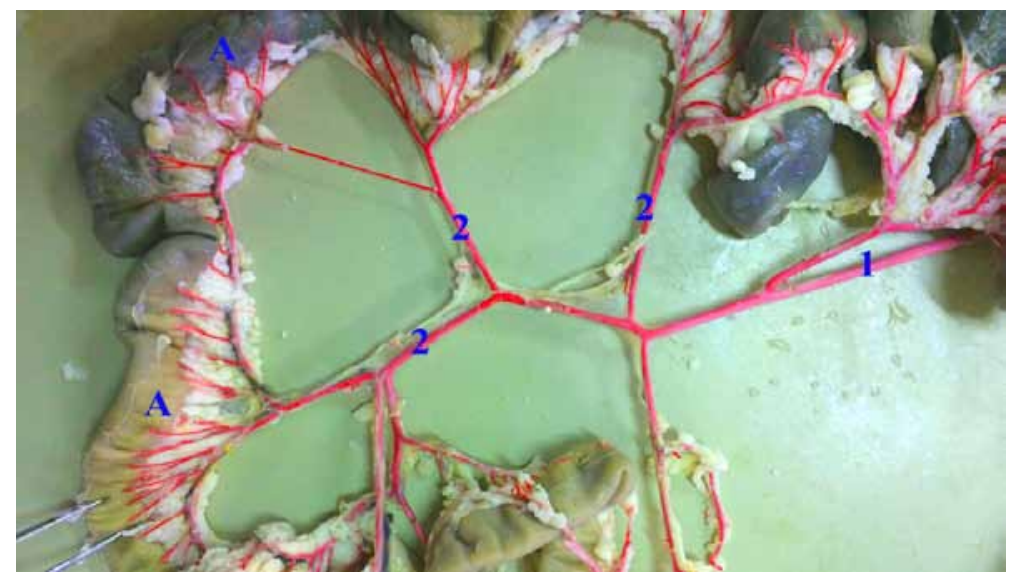

Figure 2. A photograph showing the origin; course and distribution of the jejunal arteries of the sheep; (A) Jejunum; (1) Cranial mesenteric artery; (2) Jejunal arteries.

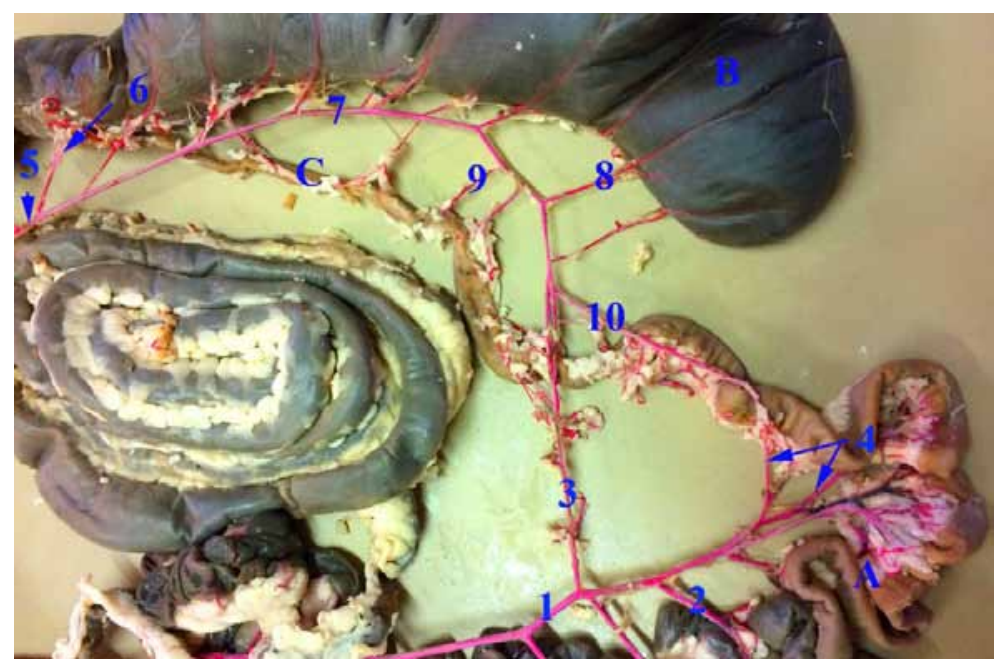

Figure 3. A photograph showing the distribution of the Antimesenteric ileal artery and ileal arteries as well as the termination of the cranial mesenteric artery of the sheep; (A) Jejunum; (B) Ileum; (C) Cecum; (1) Cranial mesenteric artery; (2) Jejunal arteries; (3) Ileal arteries; (4) Last two jejunal arteries; (5) Ileocolic artery; (6) Mesenteric ileal artery; (7) Cecal artery; (8) Cecal branches; (9) Antimesenteric ileal branches; (10) Antimesenteric ileal artery. 


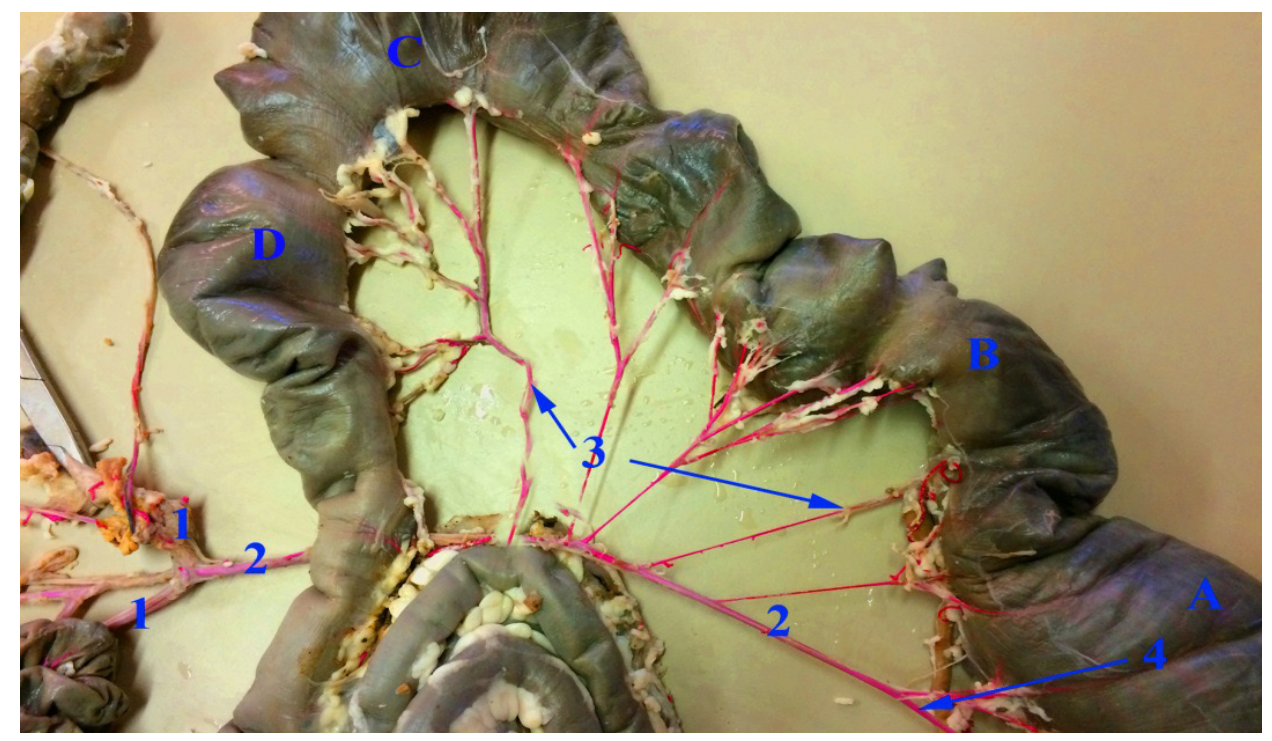

Figure 4. A photograph showing the origin; course and distribution of the colic branches of the ileocolic artery of the sheep; (A) Cecum; (B) Ventral gyrus; (C) Middle gyrus; (D) Dorsal gyrus: (1) Cranial mesenteric artery; (2) Ileocolic artery; (3) Colic branches; (4) Cecal artery.

the jejunal convolutions and divided into two main branches, cranial and caudal which anastomosed to form arches which gave several twigs were given to the jejunal wall.

\subsection{Ileal arteries}

The ileal arteries (Figures 1 and 3 ) arose from the terminal part of the cranial mesenteric artery. They passed, within the mesoileum, towards the mesenteric border of the ileum. Each ileal artery divided into a cranial and a caudal branch. They anastomosed with the last jejunal artery and the mesenteric ileal artery of the ileocolic artery.

\subsection{Ileocolic artery}

The ileocolic artery (Figures 1, 3 and 4) arose from the cranial mesenteric artery. It gave off mesenteric ileal artery (Figure 3) which followed the mesenteric aspect of the terminal part of the ileum and anastomosed with the antimesenteric ileal branches of the cecal artery and a common trunk for colic branches and right colic arteries (Figure 5) which coursed on the ansa spiralis coli toward the central flexure where it terminated by dividing into the last colic branch and the first right colic artery. This trunk gave off colic branches (Figures 4 and 5) which supplied the ansa proximalis coli, the centripetal gyri and the central flexure of ansa spiralis coli and right colic arteries (Figure 5) which supplied the centrifugal gyri and the dorsal part of the ansa distalis coli.

The cecal artery (Figures 1, 3 and 4) constituted the direct continuation of the ileocolic artery. It crossed the terminal part of the ileum to reach the ileocecal fold and extended along the ventral aspect of the cecum to its apex where it continued as antimesenteric ileal artery. Along its course, the cecal artery gave off cecal branches (Figures 1 and 3) which ran within the ileocecal fold towards the ventral aspect of the cecum and antimesenteric ileal branches (Figures 1 and 3 ) to supply the antimesenteric aspect of the ileum. The antimesenteric ileal artery (Figures 1 and 3 ) passed towards the terminal part of the jejunum to join the last jejunal branch of the cranial

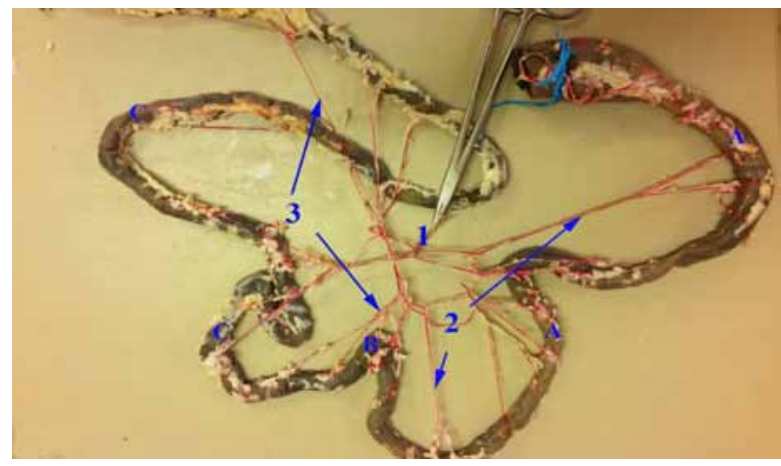

Figure 5. A photograph showing the course and distribution of the ileocolic artery of the sheep; (A) Centripetal gyri; (B) Central flexure; (C) Centrifugal gyri: (1) A common trunk for 2 and 3; (2) Colic branches; (3) Right colic arteries.

mesenteric artery. It gave off antimesenteric ileal branches to supply the antimesenteric aspect of the ileum.

\subsection{Middle colic artery}

The middle colic artery (Figure 1) coursed within the mesocolon towards the transverse colon and divided into right and left branches which supplied the ventral gyrus of the ansa distalis, transverse colon and initial part of the descending colon.

\subsection{Caudal mesenteric artery}

The caudal mesenteric artery (Figure 6) arose from the ventral aspect of the abdominal aorta, cranial to the external iliac artery. It proceeded caudoventrally within the descending mesocolon then divided into left colic artery and cranial rectal artery. The left colic artery detached colic branches and sigmoid artery, the former branches proceeded cranially along the mesenteric border of the descending colon, where it supplied its caudal two thirds by colic branches and anastomosed with the middle colic artery. While the sigmoid artery descended ventrally within the mesocolon sigmoideum to supply the 


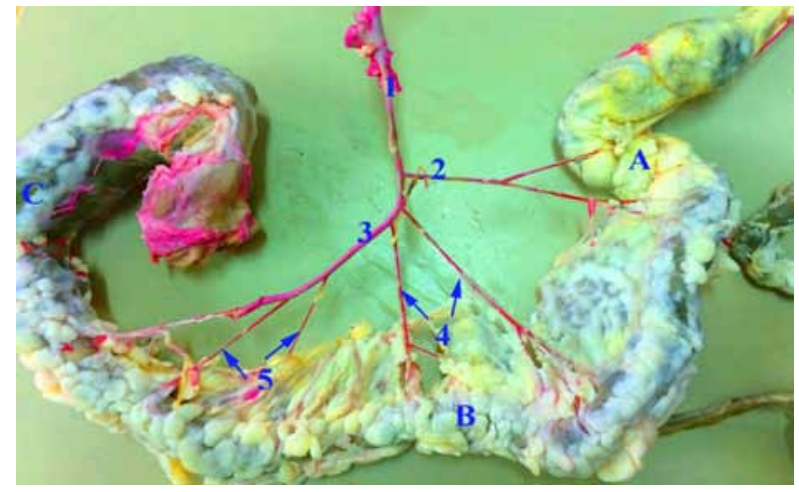

Figure 6. A photograph showing the course and distribution of the caudal mesenteric artery of the sheep; (A) Descending colon; (B) Sigmoid colon; (C) Rectum: (1) Caudal mesenteric artery; (2) Left colic artery; (3) Cranial rectal artery; (4) Sigmoid arteries; (5) Rectal branches.

sigmoid colon. While the cranial rectal artery proceeded caudally in the descending mesocolon, it gave off sigmoid arteries to the sigmoid colon then entered the pelvic cavity, where it continued in the mesorectum to the cranial segment of the rectum by rectal branches and anastomosed with the middle rectal artery.

\section{Discussion}

Gross dissection revealed that the cranial mesenteric artery in sheep originated separately from the ventral aspect of the abdominal aorta caudal to the celiac artery, the result which simulated that by Youssef (1991) in goat and König and Liebich (2011) in ruminants. While it originated by a celiaco mesenteric trunk in sheep (PEREIRA, FERREIRA and D'ERRICO, 1978), goats (FERRERIA, MIGLINO, CARNEIRO E SILVA et al., 2001), and buffaloes (MACHADO, MIGLINO, DIDIO et al., 2002).

The termination of the cranial mesenteric artery by the last two jejunal arteries given here was in accordance with that obtained by Youssef (1991) in goat. However, it terminates by the middle colic and ileocolic arteries in calves (ROOT and TASHJIAN, 1971) and by the ileocolic artery or the last 6-7 jejunal arteries in camel (WALLY, 1986; SMUTS and BEZUIDENHOUT, 1987 respectively).

Concerning the caudal duodenal artery, it was given off from the cranial mesenteric artery in the subject under investigation, thus confirming the result obtained by Youssef (1991) in goat. While, Constantinescu (2001) in goat, Constantinescu and Constantinescu (2004) in large ruminants, Machado, Miglino, Didio et al. (2002) in and Wally (1986) in camel mentioned the same origin of such artery under the name of caudal pancreaticoduodenal artery. Whereas, the artery originated from the middle colic artery in goat (NAYAR, SINGH, SINGH et al., 1983) and from the jejunal artery in camel (SMUTS and BEZUIDENHOUT, 1987).

Our findings as well as those obtained by Youssef (1991) in goat ascertained that the jejunal arteries were detached from the cranial aspect of the cranial mesenteric artery along its whole length.

There was an agreement about the origin of the ileal arteries from the terminal part of the cranial mesenteric artery in our work with that given by Ferreria, Miglino, Carneiro e Silva et al. (2001) in goat, Wilkens and Munster (1981) in ox and Machado, Miglino, Didio et al. (2002) in buffalo.

Our findings were in accordance with those gained by Youssef (1991) in goat, Wilkens and Munster (1981) as well as Simoens, De Vos and Lauvers (1981) in ruminants who said that the ileocolic artery gave off near its origin a common trunk for colic branches and right colic arteries and mesenteric ileal artery.

The ansa proximalis of the ascending colon was supplied via colic branches that arose from the ileocolic artery, while the centripetal gyri were provided with colic branches arising from the common trunk of both colic branches and right colic arteries. However, Constantinescu (2001) in goat, Constantinescu and Constantinescu (2004) in large ruminants and Machado, Miglino, Didio et al. (2002) in buffalo recorded that the colic branches of the ileocolic artery supply both ansa proximalis and centripetal gyri of the ascending colon.

Regarding the right colic arteries, they were destined to provide nutrition to the centripetal gyri and the dorsal part of the ansa distalis coli, except the most outer centrifugal gyrus which was supplied by the jejunal arteries. Similar observations were reported by Youssef (1991) in goat and Wilkens and Munster (1981) in ruminants. In this connection, Constantinescu (2001) in goat, Constantinescu and Constantinescu (2004) in large ruminants and Wally (1986) in camel stated the right colic arteries supply the ansa distalis and centrifugal gyri of the ascending colon.

Observations of the present study confirmed those of May (1970) in sheep, Youssef (1991) in goat, Maala and Sack (1981) in ox and Machado, Miglino, Didio et al. (2002) in buffalo, where the mesenteric ileal artery arose from the ileocolic artery at the ileocecal junction and followed the mesenteric border of the ileum.

The current investigation, corresponding with those of Youssef (1991) in goat, Wilkens and Munster (1981) in ruminants and Machado, Miglino, Didio et al. (2002) in buffalo, clarified that the cecal artery gave off cecal and antimesenteric ileal branches and continued as the antimesenteric ileal artery.

Regarding the middle colic artery, our findings coincided with those gained by May (1970) in sheep, Constantinescu (2001) in goat and Habel (1975) in ox, where it originated from the cranial mesenteric artery. On the contrary, such artery in camel arose from the right colic artery (WALLY, 1986).

The right and left branches of the middle colic artery were destined to supply the transverse colon and initial part of the descending colon, in addition to the ventral gyrus of the ansa distalis coli of the ascending colon. Such result was similar to that reported by May (1970) in sheep and Youssef (1991) in goat.

The caudal mesenteric artery took its origin from the ventral aspect of the abdominal aorta, confirming what given by Youssef (1991) in goat, Wilkens and Munster (1981) in small ruminants and Raghavan and Kacgroo (1964) in ox.

The obtained results were similar to those mentioned by Youssef (1991) in goat, Wilkens and Munster (1981) in ruminants and Wally (1986) in camel who reported that the left colic artery of the caudal mesenteric artery supplies the greater part of the descending colon.

The current work revealed that the cranial rectal artery arising from the caudal mesenteric artery gave off rectal branches which formed several arches to the wall of the rectum 
and anastomosed with the middle and caudal rectal arteries. Such results confirmed those given by Youssef (1991) in goat and Wilkens and Munster (1981) in ruminants.

Our observations declared that both the left colic and cranial rectal arteries detached sigmoid arteries to the sigmoid colon. Such observations were in accordance with that stated by Youssef (1991) in goat. However, the sigmoid arteries were detached from the caudal mesenteric artery itself in ruminants (WILKENS and MUNSTER, 1981), whereas they were given off from the left colic artery only in buffalo (MACHADO, MIGLINO, DIDIO et al., 2002).

\section{Conclusion}

The whole intestinal tract (except its caudal part) of the Barbados Black Belly was supplied by the branches of the cranial and caudal mesenteric arteries. The small intestine was supplied by the caudal duodenal, jejunal and ileal arteries of the cranial masseteric artery. While the large intestine was supplied by the ileocolic and middle colic arteries of the cranial mesenteric artery as well as left colic and cranial rectal arteries of the caudal mesenteric.

Acknowledgements: I am very grateful to the technical staff and lab assistances in the Department of Anatomy for their assistance.

\section{References}

CONSTANTINESCU, GM. Guide to regional ruminant anatomy based on the dissection of the goat. 1st ed. Ames: Iowa State University Press, 2001. p. 104-131.

CONSTANTINESCU, GM. and CONSTANTINESCU, IA. Clinical dissection guide for large animals, horse and large ruminants. $2 \mathrm{nd} \mathrm{ed}$. Iowa: Iowa State Press, 2004. p. 302-303.

FERRERIA, FA., MIGLINO, MA., CARNEIRO E SILVA, FO. and SANTOS, TC. Origins and ramifications of cranial and caudal mesenteric arteries in Saanen goat fetuses (Capra hircus-linnaeus, 1758). Brazilian Journal of Veterinary Research and Animal Science, 2001, vol. 38, n. 2, p. 69-73.

HABEL, RE. Abdominal aorta of ruminants. In GETTY, R. The anatomy of the domestic animals. 5 th ed. Philadelphia: W.B. Saunders, 1975. p. 982-1022.

INTERNATIONAL COMMITTEE ON VETERINARY GROSS ANATOMICAL NOMENCLATURE - ICVGAN. Nomina anatomia veterinaria. 5th ed. Columbia: Editorial Committee Hanover, 2005.
KÖNIG, HE. and LIEBICH, HG. Anatomia dos animais domésticos: texto e atlas colorido. Porto Alegre: Artmed, 2011.

MAALA, CM. and SACK, WO. The arterial supply to the ileum, cecum and the proximal loop of the ascending colon in the ox. Anatomia, Histologia, Embryologia, 1981, vol. 10, p. 130-146.

MACHADO, MRF., MIGLINO, MA., DIDIO, LJA., HONSHO, D. and BORGES, EMC. The arterial supply of buffalo intestines (Bubalus Bubalis). Buffalo Journal, 2002, vol. 18, n. 2, p. 249-256.

MAY, NS. The anatomy of the sheep. 2nd ed. Australia: University of Queen Land Press, 1970.

NAYAR, KNM., SINGH, G., SINGH, Y., SINGH, AP. and SINGH, HGR. Comparative arteriographic anatomy of the abdominal viscera and lumbar region in goats, dogs, pigs and rabbits. The Indian Journal of Animal Sciences, 1983, vol. 53, n. 12, p. 1310-1314.

PEREIRA, JGL., FERREIRA, N. and D'ERRICO, AA. Origem das artérias celíaca e mesentérica cranial, por tronco comum, em carneiros da raça Corriedale. Revista da Faculdade de Medicina Veterinária e Zootecnia da Universidade de São Paulo, 1978, vol. 15, n. 1, p. 19-22.

RAGHAVAN, D. and KACGROO, P. Anatomy of the ox. 1st ed. New Delhi: Indian Council of Agriculture Resarch, 1964. p. 530-570.

ROOT, CR. and TASHJIAN, RJ. Thoracic and abdominal arteriography in calves. American Journal of Veterinary Research, 1971, vol. 32, n. 8, p. 1193-1205. PMid:5106287.

SIMOENS, R., DE VOS, NR. and LAUVERS, H. Illustrated anatomical nomenclature of the arteries of the abdomen, the pelvis and the pelvic limb in the domestic mammals. Belgium: Laboratory of Anatomy of the Domestic Animals, Faculty of Veterinary Medicine, State University of Ghent, 1981.

SMUTS, S. and BEZUIDENHOUT, J. Anatomy of the Dromedary. Oxford: Clarendon Press, 1987. p. 156-177.

WALLY, YR. Some anatomical observations on the intestinal tract of the one humped camel (camelus dromedarius). Giza: Cairo University, 1986. [Dissertation].

WILKENS, H. and MUNSTER, W. The circulatory system. In NICKEL, A., SCHUMER, R. and SEIFERLE, E. The anatomy of the domestic animals. Hamburg: Wright Verlag Paul Parey, 1981. p. 159-268. vol. 3.

YOUSSEF, A. Some anatomical studies on the coeliac, cranial mesenteric and caudal mesenteric arteries of goat. Benha: Faculty of Veterinary Medicine, Zagazig University, 1991. [Thesis].

Received February 24, 2017 Accepted March 13, 2017 